\title{
Evaluation of fall detection classification approaches
}

\begin{abstract}
As we grow old, our desire for being independence does not decrease while our health needs to be monitored more frequently. Accidents such as falling can be a serious problem for the elderly. An accurate automatic fall detection system can help elderly people be safe in every situation. In this paper a waist worn fall detection system has been proposed. A tri-axial accelerometer (ADXL345) was used to capture the movement signals of human body and detect events such as walking and falling to a reasonable degree of accuracy. A set of laboratory-based falls and activities of daily living (ADL) were performed by healthy volunteers with different physical characteristics. This paper presents the comparison of different machine learning classification algorithms using Waikato Environment for Knowledge Analysis (WEKA) platform for classifying falling patterns from ADL patterns. The aim of this paper is to investigate the performance of different classification algorithms for a set of recorded acceleration data. The algorithms are Multilayer Perceptron, Naive Bayes, Decision tree, Support Vector Machine, ZeroR and OneR. The acceleration data with a total data of 6962 instances and 29 attributes were used to evaluate the performance of the different classification algorithm. Results show that the Multilayer Perceptron algorithm is the best option among other mentioned algorithms, due to its high accuracy in fall detection.
\end{abstract}

